\title{
Single photoeffect on helium-like ions in the non-relativistic region
}

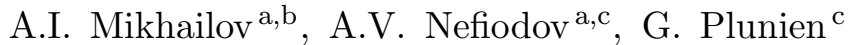 \\ a Petersburg Nuclear Physics Institute, \\ 188300 Gatchina, St. Petersburg, Russia \\ b Max-Planck-Institut für Physik komplexer Systeme, \\ Nöthnitzer Straße 38, D-01187 Dresden, Germany \\ ${ }^{\mathrm{c}}$ Institut für Theoretische Physik, Technische Universität Dresden, \\ Mommsenstraße 13, D-01062 Dresden, Germany
}

(Dated: Received November 21, 2018)

\begin{abstract}
We present a generalization of the pioneering results obtained for single K-shell photoionization of $\mathrm{H}$-like ions by M. Stobbe [Ann. Phys. 7 (1930) 661] to the case of the helium isoelectronic sequence. The total cross section of the process is calculated, taking into account the correlation corrections to first order of the perturbation theory with respect to the electron-electron interaction. Predictions are made for the entire non-relativistic energy domain. The phenomenon of dynamical suppression of correlation effects in the ionization cross section is discussed.
\end{abstract}

PACS numbers: $32.80 . \mathrm{Fb}, 32.80 .-\mathrm{t}, 31.25 . \mathrm{Eb}$ 
1. The single ionization of light atomic systems by photon impact is one of the fundamental

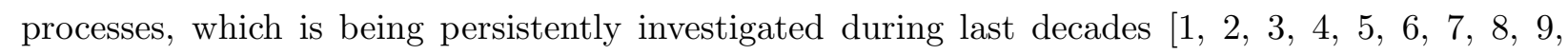
10, 11, 12]. Although the non-relativistic problem for single photoeffect on H-like atom was solved analytically by M. Stobbe already in 1930 [13], the further generalization on the case of two-electron targets, such as, for example, neutral He atom, is a non-trivial task. Since the strength of the electron-electron interaction is comparable to that of the electron-nucleus interaction, the singleparticle treatment cannot be appropriate. For theoretical calculations of ionization cross sections, it is usual to employ sophisticated numerical methods dealing with highly correlated wave functions. This allows one to take into account the electron correlations beyond the independent-particle approximation.

In this Letter, we evaluate the dominant contribution of correlation effects to the cross section for single K-shell ionization of He-like ions using the non-relativistic perturbation theory. To zeroth-order approximation, it is assumed that the atomic nucleus is the external source of the Coulomb field, while the interaction of electrons with each other is neglected. The non-interacting electrons are described by the Coulomb wave functions for the discrete and continuous spectra (Furry picture). The electron-electron interaction is treated within the framework of perturbation theory. The latter exhibits fast convergence even for small values of nuclear charge $Z$ [14]. To first order of perturbation theory, the problem of single ionization of He-like ions is reduced to the evaluation of one-photon exchange diagrams. The correlation corrections to the cross section arise due to modification of the binding energy and wave functions of the initial and final states. Using this approach, we have already deduced the universal scaling behavior for the ionization cross section at high photon energies [15]. The formula obtained previously has a simple analytical form, but it is valid only in the asymptotic non-relativistic limit. This Letter reports on the extension of results of our work [15] on the entire non-relativistic energy domain.

2. The non-relativistic problem of single ionization of a K-shell bound electron by photon impact involves the following quantities: the momentum $\boldsymbol{k}$, the energy $\omega=|\boldsymbol{k}|=k$, and the polarization vector e of an incident photon, the binding energy $I=\eta^{2} /(2 m)$ and the average momentum $\eta=m \alpha Z$ of a K-shell electron, where $m$ is the electron mass and $\alpha$ is the fine-structure constant $(\hbar=1, c=1)$. The outgoing electron is characterized by the energy $E_{p}=p^{2} /(2 m)$ and the momentum $\boldsymbol{p}$ at infinity. We shall employ the Coulomb gauge, in which $(\mathbf{e} \cdot \boldsymbol{k})=0$ and $\left(\mathbf{e}^{*} \cdot \mathbf{e}\right)=1$. The parameter $\alpha Z$ is supposed to be sufficiently small, that is, $\alpha Z \ll 1$.

The amplitude for single photoeffect on a bound K-shell electron is described by the Feynman diagram depicted in Fig. 1(a). The energy-conservation law reads $E_{p}=\omega-I$. Accordingly, the 
non-relativistic photoionization can proceed at the photon energies $I \leqslant \omega \ll m$. Using the oneelectron Coulomb wave functions for the discrete and continuous spectra, Stobbe has obtained an analytical expression for the cross section of single K-shell photoeffect, which can be cast into the following form [13, 16]

$$
\sigma_{\mathrm{K}}^{+}=\alpha a_{0}^{2} \frac{2^{9} \pi^{2}}{3 Z^{2}} \frac{\exp \left(-4 \xi \cot ^{-1} \xi\right)}{\left(1+\xi^{-2}\right)^{4}[1-\exp (-2 \pi \xi)]},
$$

where $a_{0}=1 /(m \alpha)$ is the Bohr radius. In Eq. (11), we have introduced the dimensionless parameter $\xi=\eta / p$. The quantity $\xi^{-1}$ has the meaning of the momentum $p$ of the outgoing electron, which is calibrated in units of the characteristic momentum $\eta$. Due to the energy-conservation law, $\xi$ is related with the dimensionless energy of the photon $\varepsilon_{\gamma}=\omega / I$ according to $\xi=1 / \sqrt{\varepsilon_{\gamma}-1}$ or, equivalently, $\xi=1 / \sqrt{\varepsilon_{p}}$, where $\varepsilon_{p}=E_{p} / I$ is the dimensionless energy of the outgoing electron. Equation (1) is valid in the entire non-relativistic energy domain $1 \leqslant \varepsilon_{\gamma} \ll 2(\alpha Z)^{-2}$. In the derivation, the dipole approximation has been employed, which implies that the photon momentum $\boldsymbol{k}$ is negligible compared with the electron momentum $\boldsymbol{p}$.

In the non-relativistic photoeffect, one is often interested in the asymptotic energy domain far beyond the threshold, which is characterized by $1 \ll \varepsilon_{\gamma} \ll 2(\alpha Z)^{-2}$. In this case, Eq. (11) can be expanded with respect to the small parameter $\xi \ll 1$. The leading term in the $\xi$ expansion is given by [16]

$$
\sigma_{\mathrm{K}}^{+}=\alpha a_{0}^{2} \frac{2^{8} \pi}{3 Z^{2}} \varepsilon_{\gamma}^{-7 / 2}, \quad(\xi \ll 1) .
$$

In fact, Eq. (2) is obtained using the Born approximation, which describes the wave function of the outgoing electron in terms of a plane wave. Since Eq. (11) involves also the parameter $\pi \xi$, which originates from the normalization factor of the Coulomb wave function of the continuous spectrum, the convergence of the $\xi$ expansion is sufficiently slow.

Another limit of expression (11) corresponds to the ionization threshold $\left(\varepsilon_{\gamma} \rightarrow 1\right)$. In this case, the cross section reaches the constant value [16]

$$
\sigma_{\mathrm{K}}^{+}=\alpha a_{0}^{2} \frac{2^{9} \pi^{2}}{3 e^{4} Z^{2}}, \quad(\xi \gg 1),
$$

where $e \simeq 2.71828$ is the Napier-Euler number.

3. To zeroth-order approximation, the amplitude $\mathcal{A}^{(0)}$ for the single photoeffect on two-electron atomic system in the ground state is described by the Feynman graph depicted in Fig. 1(b). In 
the momentum representation, it can be written as follows

$$
\begin{aligned}
\mathcal{A}^{(0)} & =\sqrt{2} C \frac{\exp \left(-2 \xi \cot ^{-1} \xi\right)}{\left(1+\xi^{2}\right)^{2}}, \\
C & =8 \pi \eta N_{\gamma} N_{1 s} N_{p}(1-i \xi) \frac{(\mathbf{e} \cdot \boldsymbol{p})}{p^{4}}, \\
N_{\gamma}^{2} & =\frac{4 \pi \alpha}{\eta^{2} \varepsilon_{\gamma} m}, \quad N_{1 s}^{2}=\frac{\eta^{3}}{\pi}, \\
N_{p}^{2} & =\frac{2 \pi \xi}{1-\exp (-2 \pi \xi)} .
\end{aligned}
$$

Here $\xi=\eta / p$ and the dipole approximation $(k=0)$ has been again used in the derivation. The corresponding expression for the total cross section $\sigma_{0}^{+}$of the process reads

$$
\sigma_{0}^{+}=2 \sigma_{\mathrm{K}}^{+}
$$

where $\sigma_{\mathrm{K}}^{+}$is given by Eq. (11). The factor 2 accounts for the number of electrons in the target. The energy-conservation law keeps the same form as for the H-like ion, namely, $\varepsilon_{p}=\varepsilon_{\gamma}-1$. Equation (6) is valid in the entire non-relativistic domain $1 \leqslant \varepsilon_{\gamma} \ll 2(\alpha Z)^{-2}$. However, in the case of light two-electron atoms, the expression (6) is a quite rough approximation, because the electronelectron interaction is completely neglected. For example, for the neutral He atom, Eq. (6) predicts $\sigma_{0}^{+}=3.15 \mathrm{Mb}$ at the ionization threshold $(\xi \rightarrow \infty)$. The corresponding experimental value turns out to be $\sigma_{\exp }^{+}=7.40 \mathrm{Mb}$ at the photon energy $\omega=24.59 \mathrm{eV}$ [7]. The significant discrepancy between these results is due to correlation effects. Near the threshold, the approximation of noninteracting electrons strongly underestimates the experimental cross section. On the contrary, in the asymptotic high-energy limit, Eq. (6) yields an upper bound for the photoionization cross section [15].

Now we shall take into account the electron-electron interaction using the perturbation theory. To first order, the amplitude $\mathcal{A}^{(1)}$ for the single K-shell photoeffect on He-like ion is described by the gauge invariant set of four Feynman graphs depicted in Fig. 2. The total amplitude for the process is given by $\mathcal{A} \simeq \mathcal{A}^{(0)}+\mathcal{A}^{(1)}$, where the amplitude (4) should be calculated with taking into account the correlation correction to the binding energy. More specifically, the energy-conservation law now implies $\varepsilon_{p}=\varepsilon_{\gamma}-1+\delta_{1}$, where $\delta_{1}=\Delta_{1} / Z$ is the correction to the binding energy of the ground state calculated to first order of the perturbation theory and $\Delta_{1}=5 / 4$ [17]. In calculations of the amplitude $\mathcal{A}^{(1)}$, the correlation correction $\delta_{1}$ to the binding energy can be omitted, because it exceeds the desired level of accuracy.

Note, that within the asymptotic high-energy domain, the binding energies are assumed to be negligibly small with respect to the photon energies. In addition, the wave function of the outgoing 
electron can be approximated by the plane wave, so that the Feynman graph in Fig. 2(a) yields the dominant contribution to the total cross section for single photoionization, provided the Coulomb gauge is employed [15]. However, for low energies, all Feynman diagrams depicted in Fig. 2 are expected to contribute with the same order of magnitude, while the plane-wave approximation becomes inadequate.

Since the general method for evaluation of the Coulomb matrix elements has been already described in more details [18], we focus here on presentation of the explicit expression for the amplitude $\mathcal{A}^{(1)}=\sum_{\beta} \mathcal{A}_{\beta}^{(1)}$ only. The individual contributions of each diagram in Figs. 2(a)-(d) read

$$
\begin{aligned}
& \mathcal{A}_{\mathrm{a}}^{(1)}=-\frac{\sqrt{2}}{8} \frac{C}{Z}\left\{\left(\frac{10}{1+\xi^{2}}+\frac{3}{4}-3 \ln 2\right) f(0)+\right. \\
& \left.+\left(\frac{1}{2}+3 \ln 2\right) f(1 / 2)+3 \int_{0}^{1 / 2} d x f^{\prime}(x) \ln x\right\} \\
& \mathcal{A}_{\mathrm{b}}^{(1)}=-i \sqrt{2} \xi \frac{C}{Z} \int_{0}^{1} \frac{d x}{\Lambda} x^{1-i \xi}\left(\frac{1+\Lambda}{1+i \xi}\right)^{2 i \xi}\left\{\Phi_{\lambda}-\Phi_{\nu}+\xi \frac{\partial \Phi_{\nu}}{\partial \nu}\right\}_{\substack{\nu=2 \xi \\
\lambda \rightarrow 0}}, \\
& \mathcal{A}_{\mathrm{c}}^{(1)}=8 \sqrt{2} \xi^{4} \frac{C}{Z} \int_{0}^{1} \frac{d x}{u} \frac{x^{1-\xi / \mu}}{(u+\xi)^{2}}\left(\frac{u+\mu}{\xi+\mu}\right)^{2 \xi / \mu} \times \\
& \times \int_{0}^{1} \frac{d y}{\chi^{4}} \sqrt{y}\left\{\frac{\xi}{2} \chi+v(\xi+2 v)(2 \xi+3 v)\right\} e^{-2 \xi \cot ^{-1}(\xi+v)}, \\
& \mathcal{A}_{\mathrm{d}}^{(1)}=2 \sqrt{2} \xi^{4} \frac{C}{Z} \frac{\partial^{2}}{\partial \zeta \partial \rho} \int_{0}^{1} \frac{d x}{\Lambda} x^{1-i \xi}\left(\frac{1+\Lambda}{1+i \xi}\right)^{2 i \xi} \times \\
& \times\left.\int_{0}^{1} \frac{d y y}{Q} \frac{\left(1-(Q+i \rho)^{2}\right)^{i \xi-2}}{\left(-(1+Q+i \rho)^{2}\right)^{i \xi}}\right|_{\substack{\zeta=\xi \\
\rho=\xi}}, \\
& f(x)=\frac{\left(1-x^{2}\right) \exp \left[-2 \xi \cot ^{-1}(\tau \xi)\right]}{\left[(1-x)^{2}+\xi^{2}(1+x)^{2}\right]^{2}}, \quad \tau=\frac{1+x}{1-x}, \\
& \Phi_{\lambda}=\frac{\left(1-(\Lambda+i \lambda)^{2}\right)^{i \xi-2}}{\left(-(1+\Lambda+i \lambda)^{2}\right)^{i \xi}}, \quad \Lambda=\sqrt{1-x\left(1+\xi^{2}\right)}, \\
& u=\sqrt{1-x+\xi^{2}(2-x)}, \quad v=\sqrt{y}(u+\xi), \quad \mu=\sqrt{1+2 \xi^{2}}, \\
& \chi=1+(\xi+v)^{2}, \quad Q=\sqrt{y}(\Lambda+i \zeta) .
\end{aligned}
$$

Here the function $C$ is defined by Eq. (5). In the integral of Eq. (7), the prime at the function $f(x)$ denotes the derivative with respect to the variable $x$. We note, that the matrix element corresponding to the graph in Fig. 2(a) involves the reduced Green's function. This requires isolation and cancelation of the pole term, which has been done analytically. In Eq. (8), after 
taking the derivative with respect to $\nu$, one should set $\nu=2 \xi$, where $\xi=\eta / p$. In addition, the parameter $\lambda$ of a Yukawa-type screened Coulomb interaction introduced for regularization of the integral should tend to zero. In Eq. (10), after taking the derivatives with respect to $\zeta$ and $\rho$, one should set $\zeta=\rho=\xi$. Note also that the contribution of the graph in Fig. 2(b) describing the finalstate interaction, contains a logarithmically divergent term $\ln (\lambda / 2 p)$ for $\lambda \rightarrow 0$. However, this term drops out in the expression for cross section of the process. This fact reflects a general property of the wave function for continuous spectrum, when it is constructed using perturbation theory with respect to the Coulomb interaction. More precisely, the logarithmically divergent terms of the wave function can be summed up to an overall phase factor $\exp [i \xi \ln (\lambda / 2 p)]$, which does not contribute to the cross section [19, 20, 21, 22, 23].

Separating explicitly the contributions due correlation corrections to the binding energy and wave functions, the cross section for single K-shell photoionization of He-like ions can be cast into the following form

$$
\sigma^{+}=\sigma_{0}^{+}\left\{1+\left[\frac{\Delta_{1}}{\varepsilon_{\gamma}}+a_{1}(\xi)\right] \frac{1}{Z}\right\}
$$

where $\sigma_{0}^{+}$is given by Eq. (하), $\xi=1 / \sqrt{\varepsilon_{p}}, \varepsilon_{p}=\varepsilon_{\gamma}-1+\delta_{1}, \delta_{1}=\Delta_{1} / Z$, and $\Delta_{1}=5 / 4$. The quantity $a_{1}(\xi)=2 Z \sum_{\beta} \operatorname{Re}\left(\mathcal{A}_{\beta}^{(1)} / \mathcal{A}^{(0)}\right)$ is a universal function of the parameter $\xi$ (see Fig. 3 and Table 【). Equation (11) is valid in entire non-relativistic domain $1-\delta_{1} \leqslant \varepsilon_{\gamma} \ll 2(\alpha Z)^{-2}$ and represents the main result of this Letter.

The function $a_{1}(\xi)$ describes the "dynamical correlation", originating from the correlated twoelectron wave functions both in the initial and final states. Three characteristic domains can be distinguished here, namely, the threshold domain $(\xi \gg 1)$, the transition domain $(\xi \simeq 1)$, and the high-energy domain $(\xi \ll 1)$. As can be seen from Fig. 3, for values $\xi \lesssim 10^{-1}$ and $\xi \gtrsim 10$, the function $a_{1}(\xi)$ saturates rapidly approaching the constant limits, while it undergoes significant changes within the range $10^{-1} \lesssim \xi \lesssim 10$. Within the threshold domain, the consistent account of the correlation interaction both in the initial and final states becomes especially crucial. In the asymptotic high-energy limit, $a_{1}(\xi)$ tends to the analytical value [15]

$$
a_{1}=-\frac{19}{16}+\frac{3}{4} \ln 2 \simeq-0.6676
$$

while the correlation correction in the binding energy is negligible for the cross section. In this case, the correlation effect results predominantly from the wave functions. In particular, within the Coulomb gauge, this is just the contribution from the wave function of the initial state. At about $\xi \simeq 0.6036$, the function $a_{1}(\xi)$ tends to zero. Accordingly, the correlation effect in the cross section arises due to the binding energy. 
For some values of the parameter $\xi$, the correlation term in the square brackets in Eq. (11) vanishes. In this case, the correlation effect due to the binding energy cancels with that due to the wave functions. As a result, the cross section (11) coincides with the Coulomb prediction (6). In Table II, we consider the particular examples, where the dynamical suppression of correlation effects take place. Note, that for neutral helium atom the interpolation of experimental cross sections predicted in Ref. 7] yields $\sigma_{\exp }^{+}=14.2 \mathrm{~kb}$ at the photon energy $\omega=308.2 \mathrm{eV}$. The deviation from the corresponding theoretical value $\sigma_{0}^{+}=15.6 \mathrm{~kb}$ is exclusively due to higher-order correlation corrections neglected in the present consideration.

In Table III, we present a comparison between our predictions according to Eq. (11) for several two-electron targets at different photon energies with the numerical calculations of the work [2]. Bell and Kingston used the Hartree-Fock wave functions for the continuum state and many-parametrical variational wave functions for the ground state. The results of Ref. [2] are slightly gauge dependent. For very light targets, such as neutral He atom, the account for the correlation correction $\delta_{1}$ to the binding energy is still not sufficient to reproduce the experimental thresholds. Certainly, one needs to go beyond the first-order approximation. However, although consistent calculations of the binding energies have been performed with taking into account of two- and three-photon exchange diagrams [24, 25], the evaluation of ionization cross sections at the same level of accuracy seems to be not feasible in the near future. Accordingly, in Table III, we merely employ the experimental threshold energies $\omega$. As can be seen, the formula (11) turns out to be in good agreement with the numerical results by Bell and Kingston [2] both at the ionization threshold and beyond.

\section{Acknowledgments}

A.M. is grateful to the Dresden University of Technology for the hospitality and for financial support from Max Planck Institute for the Physics of Complex Systems. A.N. and G.P. acknowledge financial support from DFG, BMBF, and GSI. This research has been also supported by RFBR (Grant no. 05-02-16914) and INTAS (Grant no. 06-1000012-8881). 
TABLE I: For various values of the dimensionless parameter $\xi$, the universal quantities $a_{1}(\xi)$ are tabulated. Numbers in parentheses indicate powers of 10 .

\begin{tabular}{cccccccccccccc}
\hline$\xi$ & $a_{1}(\xi)$ & $\xi$ & $a_{1}(\xi)$ & $\xi$ & $a_{1}(\xi)$ & $\xi$ & $a_{1}(\xi)$ & $\xi$ & $a_{1}(\xi)$ & $\xi$ & $a_{1}(\xi)$ & $\xi$ & $a_{1}(\xi)$ \\
\hline $1.0(-3)$ & -0.6676 & 0.2 & -0.5362 & 0.7 & 0.1099 & 1.2 & 0.4822 & 1.7 & 0.6448 & 4.0 & 0.8048 & 30 & 0.8433 \\
$0.5(-2)$ & -0.6675 & 0.3 & -0.4087 & 0.8 & 0.2097 & 1.3 & 0.5262 & 1.8 & 0.6645 & 5.0 & 0.8190 & 50 & 0.8438 \\
$1.0(-2)$ & -0.6672 & 0.4 & -0.2693 & 0.9 & 0.2955 & 1.4 & 0.5634 & 1.9 & 0.6816 & 7.0 & 0.8313 & 80 & 0.8439 \\
$0.5(-1)$ & -0.6580 & 0.5 & -0.1319 & 1.0 & 0.3684 & 1.5 & 0.5949 & 2.0 & 0.6965 & 10 & 0.8378 & $1.0(2)$ & 0.8439 \\
0.1 & -0.6309 & 0.6 & -0.0043 & 1.1 & 0.4302 & 1.6 & 0.6218 & 3.0 & 0.7763 & 20 & 0.8424 & $1.0(3)$ & 0.8440 \\
\hline
\end{tabular}

TABLE II: For different values of the nuclear charge $Z$, the parameters $\xi$, the dimensionless energies $\varepsilon_{\gamma}$, the Coulomb ionization potentials $I$, the photon energies $\omega$, and the corresponding ionization cross sections $\sigma^{+}$are tabulated. For the considered values of $\xi$, the predictions according to Eq. (11) coincide with those according to Eq. (6).

\begin{tabular}{lccccccccccc}
\hline$Z$ & $\xi$ & $\varepsilon_{\gamma}$ & $I(\mathrm{eV})$ & $\omega(\mathrm{eV})$ & $\sigma^{+}(\mathrm{kb})$ & $Z$ & $\xi$ & $\varepsilon_{\gamma}$ & $I(\mathrm{eV})$ & $\omega(\mathrm{keV})$ & $\sigma^{+}(\mathrm{kb})$ \\
\hline 2 & 0.4348 & 5.663 & 54.42 & 308.2 & 15.6 & 7 & 0.4422 & 5.936 & 666.7 & 3.957 & 1.39 \\
3 & 0.4383 & 5.789 & 122.4 & 708.8 & 7.24 & 8 & 0.4425 & 5.950 & 870.8 & 5.181 & 1.07 \\
4 & 0.4400 & 5.853 & 217.7 & 1274 & 4.15 & 9 & 0.4428 & 5.961 & 1102 & 6.569 & 0.847 \\
5 & 0.4410 & 5.891 & 340.1 & 2004 & 2.69 & 10 & 0.4430 & 5.970 & 1361 & 8.122 & 0.688 \\
6 & 0.4417 & 5.917 & 489.8 & 2898 & 1.88 & 11 & 0.4432 & 5.977 & 1646 & 9.840 & 0.570 \\
\hline
\end{tabular}


TABLE III: For various two-electron targets, the nuclear charges $Z$, the photon energies $\omega$, the corresponding dimensionless energies $\varepsilon_{\gamma}$, and the photoionization cross sections $\sigma^{+}$are tabulated. Our predictions are made according to Eq. (11). The calculations by Bell and Kingston [2] have been performed using different gauges: velocity (a) and length (b). Numbers in parentheses indicate powers of 10.

\begin{tabular}{|c|c|c|c|c|c|c|c|c|c|c|c|}
\hline \multirow[t]{2}{*}{ Target } & \multirow{2}{*}{\multicolumn{2}{|c|}{$Z \omega(\mathrm{eV})$}} & \multirow[t]{2}{*}{$\varepsilon_{\gamma}$} & \multicolumn{3}{|c|}{$\sigma^{+}(\mathrm{Mb})$} & \multirow[t]{2}{*}{$\omega(\mathrm{keV})$} & \multirow[t]{2}{*}{$\varepsilon_{\gamma}$} & \multicolumn{3}{|c|}{$\sigma^{+}(\mathrm{b})$} \\
\hline & & & & Eq. (11) & Ref. $[2]^{\mathrm{a}}$ & Ref. $[2]^{\mathrm{b}}$ & & & Eq. (11) & Ref. $[2]^{\mathrm{a}}$ & Ref. $[2]^{\mathrm{b}}$ \\
\hline \multirow[t]{5}{*}{$\mathrm{He}$} & 2 & 24.59 & 0.452 & 7.19 & 7.15 & 7.32 & 0.456 & 8.375 & $4.68(3)$ & $4.40(3)$ & $4.80(3)$ \\
\hline & & 61.23 & 1.125 & 1.21 & 1.07 & 1.14 & 0.891 & 16.37 & 553 & 527 & 558 \\
\hline & & 183.7 & 3.375 & $7.19(-2)$ & $6.64(-2)$ & $7.34(-2)$ & 1.327 & 24.37 & 151 & 146 & 151 \\
\hline & & 238.1 & 4.375 & $3.39(-2)$ & $3.15(-2)$ & $3.47(-2)$ & 1.762 & 32.37 & 59.5 & 57.4 & 59.0 \\
\hline & & 346.9 & 6.375 & $1.09(-2)$ & $1.02(-2)$ & $1.12(-2)$ & 3.503 & 64.37 & 6.05 & 5.74 & 6.12 \\
\hline \multirow[t]{5}{*}{$\mathrm{Li}^{+}$} & 3 & 75.64 & 0.618 & 2.49 & 2.57 & 2.61 & 0.289 & 2.361 & $9.88(4)$ & $9.56(4)$ & $9.96(4)$ \\
\hline & & 112.2 & 0.917 & 1.07 & 1.02 & 1.05 & 0.398 & 3.250 & $4.01(4)$ & $3.88(4)$ & $4.06(4)$ \\
\hline & & 153.1 & 1.250 & $5.19(-1)$ & $4.98(-1)$ & $5.11(-1)$ & 1.378 & 11.25 & 918 & 897 & 928 \\
\hline & & 180.3 & 1.472 & $3.45(-1)$ & $3.33(-1)$ & $3.43(-1)$ & 1.813 & 14.81 & 383 & 375 & 386 \\
\hline & & 234.7 & 1.917 & $1.74(-1)$ & $1.68(-1)$ & $1.74(-1)$ & 3.554 & 29.03 & 43.2 & 41.7 & 44.0 \\
\hline \multirow[t]{5}{*}{$\overline{\mathrm{B}^{3+}}$} & 5 & 259.3 & 0.762 & 0.730 & 0.743 & 0.748 & 0.473 & 1.390 & $1.67(5)$ & $1.65(5)$ & $1.67(5)$ \\
\hline & & 295.9 & 0.870 & 0.537 & 0.528 & 0.533 & 0.582 & 1.710 & $9.65(4)$ & $9.53(4)$ & $9.64(4)$ \\
\hline & & 336.7 & 0.990 & 0.393 & 0.388 & 0.391 & 1.126 & 3.310 & $1.50(4)$ & $1.48(4)$ & $1.51(4)$ \\
\hline & & 363.9 & 1.070 & 0.325 & 0.321 & 0.323 & 1.561 & 4.590 & $5.72(3)$ & $5.64(3)$ & $5.74(3)$ \\
\hline & & 418.4 & 1.230 & 0.229 & 0.226 & 0.228 & 3.738 & 10.99 & 388 & 384 & 399 \\
\hline \multirow[t]{5}{*}{$\mathrm{O}^{6+}$} & 8 & 739.0 & 0.849 & 0.251 & 0.253 & 0.254 & 3.782 & 4.344 & $2.77(3)$ & $2.76(3)$ & $2.78(3)$ \\
\hline & & 1497 & 1.719 & $3.99(-2)$ & $3.98(-2)$ & $4.00(-2)$ & 5.306 & 6.094 & 994 & 990 & 996 \\
\hline & & 1878 & 2.156 & $2.13(-2)$ & $2.12(-2)$ & $2.13(-2)$ & 6.830 & 7.844 & 457 & 455 & 458 \\
\hline & & 2259 & 2.594 & $1.26(-2)$ & $1.25(-2)$ & $1.26(-2)$ & 9.878 & 11.34 & 144 & 144 & 144 \\
\hline & & 3020 & 3.469 & $5.40(-3)$ & $5.38(-3)$ & $5.41(-3)$ & 12.92 & 14.84 & 61.4 & 61.1 & 61.4 \\
\hline \multirow{5}{*}{\multicolumn{2}{|c|}{$\mathrm{Ne}^{8+}$}} & 1195 & 0.878 & 0.153 & 0.154 & 0.155 & 7.068 & 5.195 & $1.05(3)$ & $1.05(3)$ & $1.05(3)$ \\
\hline & & 1313 & 0.965 & 0.121 & 0.121 & 0.121 & 9.027 & 6.635 & 498 & 497 & 498 \\
\hline & & 2660 & 1.955 & $1.83(-2)$ & $1.83(-2)$ & $1.83(-2)$ & 12.95 & 9.515 & 163 & 162 & 163 \\
\hline & & 4129 & 3.035 & $5.20(-3)$ & $5.19(-3)$ & $5.21(-3)$ & 16.86 & 12.39 & 70.9 & 70.7 & 70.9 \\
\hline & & 5109 & 3.755 & $2.78(-3)$ & $2.77(-3)$ & $2.79(-3)$ & 24.46 & 17.97 & 21.7 & 21.4 & 21.5 \\
\hline
\end{tabular}




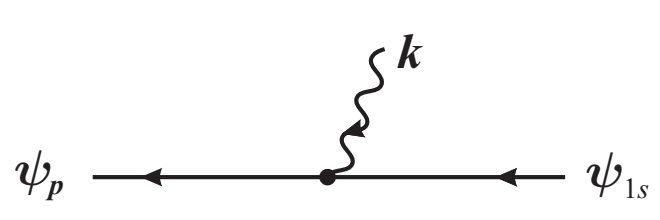

(a)
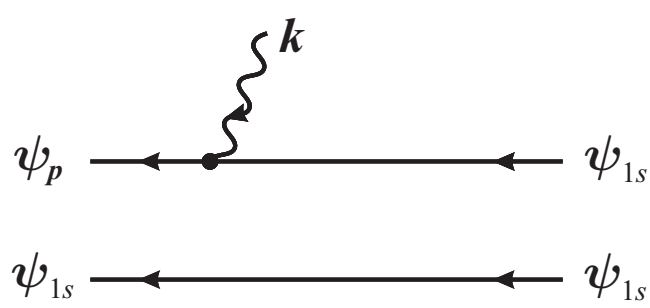

(b)

FIG. 1: Feynman diagrams for the single ionization of a K-shell electron by a single photon. Diagram (a), hydrogen-like ion; diagram (b), helium-like ion without taking into account the electron-electron interaction.

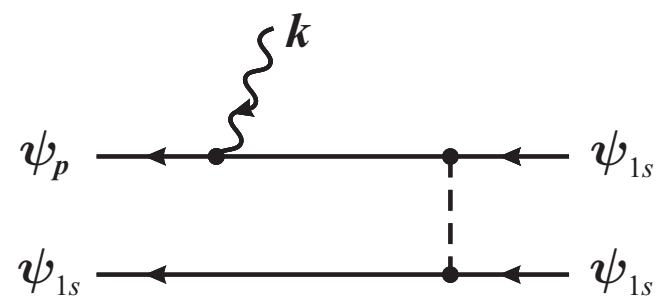

(a)

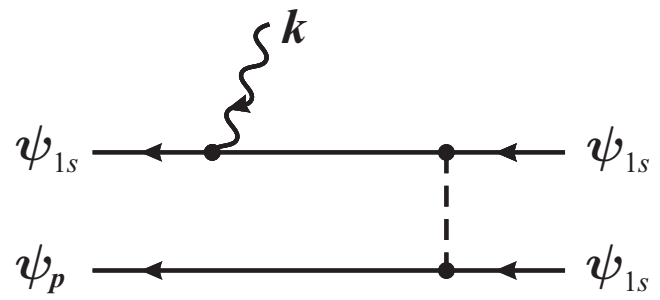

(c)

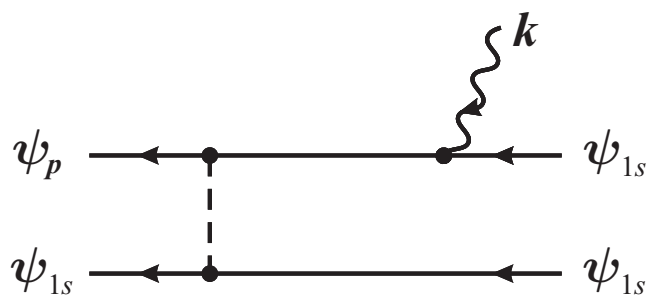

(b)

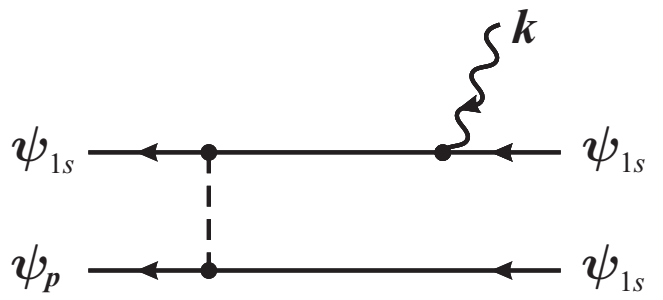

(d)

FIG. 2: Feynman diagrams for the single K-shell photoionization of He-like ion. Diagrams (a) and (c) take into account the electron-electron interaction in the initial state, while diagrams (b) and (d) account for it in the final state. The individual contributions of each diagram are gauge dependent, while total contribution of all diagrams is gauge independent. 


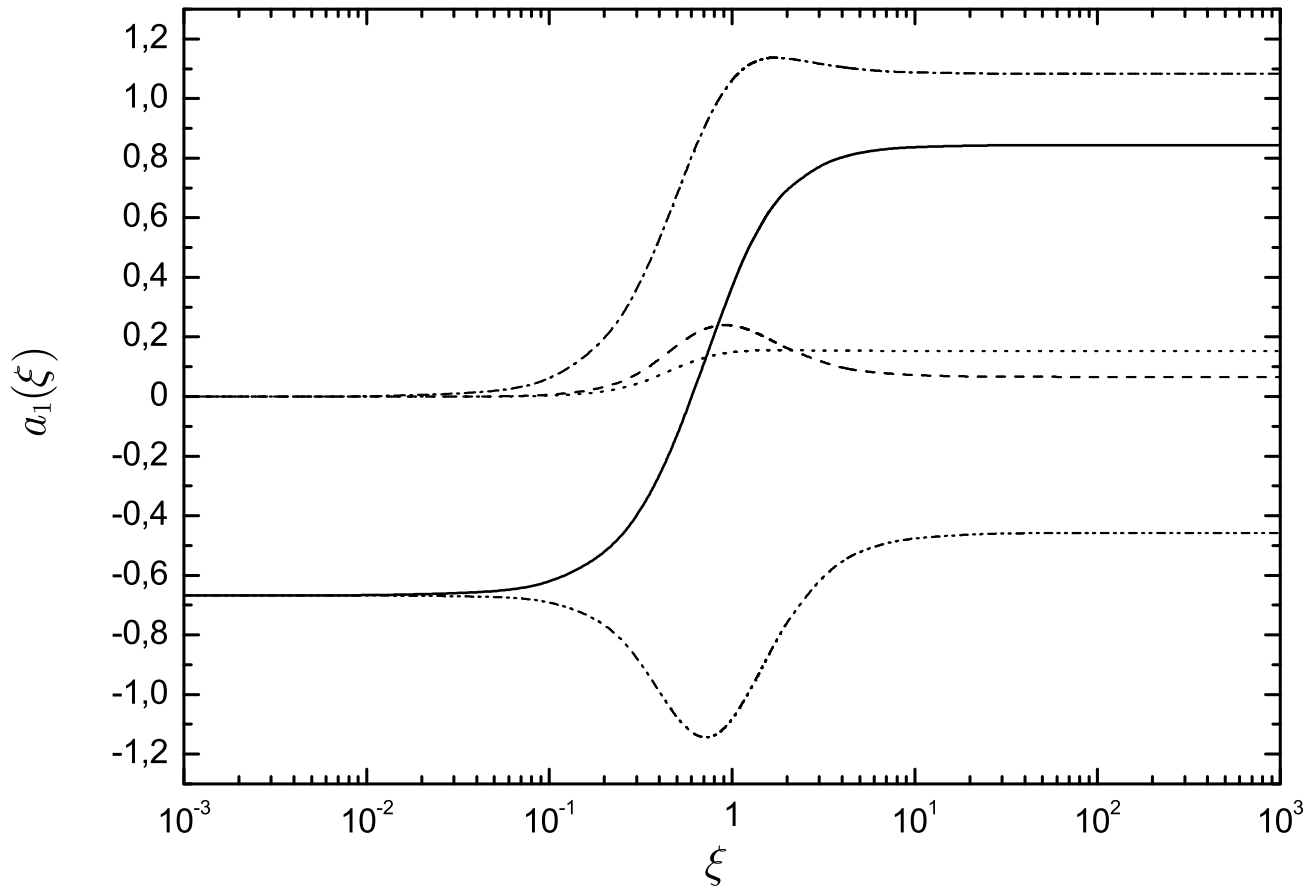

FIG. 3: Different contributions to the universal function $a_{1}(\xi)$ calculated in Coulomb gauge. Dash-double dotted line, contribution due to the diagram in Fig. 2(a); dash-dotted line, contribution due to the diagram in Fig. 2(b); dotted line, contribution due to the diagram in Fig. 2(c); dashed line, contribution due to the diagram in Fig. 2(d); solid line, total contribution of all diagrams.

[1] K.L. Bell, A.E. Kingston, J. Phys. B 3 (1970) 1433.

[2] K.L. Bell, A.E. Kingston, J. Phys. B 4 (1971) 1308.

[3] K.L. Bell, A.E. Kingston, J. Phys. B 5 (1972) 517.

[4] K.L. Bell, A.E. Kingston, I.R. Taylor, J. Phys. B 6 (1973) 1228.

[5] J.G. Cordes, M.G. Calkin, J. Phys. B 13 (1980) 4111.

[6] J.A. Fernley, K.T. Taylor, M.J. Seaton, J. Phys. B 20 (1987) 6457.

[7] J.A.R. Samson, Z.X. He, L. Yin, G.N. Haddad, J. Phys. B 27 (1994) 887.

[8] T. Surić, E.G. Drukarev, R.H. Pratt, Zh. Eksp. Teor. Fiz. 124 (2003) 243, JETP 97 (2003) 217.

[9] T. Surić, E.G. Drukarev, R.H. Pratt, Phys. Rev. A 67 (2003) 022709; ibid. 67 (2003) 059902(E).

[10] T. Surić, R.H. Pratt, J. Phys. B 37 (2004) L93.

[11] T. Surić, Rad. Phys. Chem. 70 (2004) 253.

[12] N.A.B. Faye, A.S. Ndao, A. Konte, M. Biaye, A. Wague, Phys. Scr. 71 (2005) 602. 
[13] M. Stobbe, Ann. Phys. 7 (1930) 661.

[14] A.I. Mikhailov, I.A. Mikhailov, A.V. Nefiodov, G. Plunien, G. Soff, Pis'ma Zh. Eksp. Teor. Fiz. 78 (2003) 141, JETP Lett. 78 (2003) 110.

[15] A.I. Mikhailov, A.V. Nefiodov, G. Plunien, Phys. Lett. A 358 (2006) 211.

[16] V.B. Berestetskii, E.M. Lifshits, L.P. Pitaevskii, Quantum Electrodynamics (2nd ed.), Pergamon Press, London, 1982.

[17] L.D. Landau, E.M. Lifshits, Quantum Mechanics: Non-relativistic Theory (3rd ed.), Pergamon Press, London, 1977.

[18] A.I. Mikhailov, I.A. Mikhailov, A.N. Moskalev, A.V. Nefiodov, G. Plunien, G. Soff, Phys. Rev. A 69 (2004) 032703.

[19] V.G. Gorshkov, Zh. Eksp. Teor. Fiz. 40 (1961) 1481, Sov. Phys. JETP 13 (1961) 1037.

[20] J.R. Taylor, Nuovo Cim. B 23 (1974) 313.

[21] J. McEnnan, L. Kissel, R.H. Pratt, Phys. Rev. A 13 (1976) 532; ibid. 13 (1976) 2325(E).

[22] M. Kolsrud, J. Phys. A 11 (1978) 1271.

[23] A.I. Mikhailov, Zh. Eksp. Teor. Fiz. 98 (1990) 838.

[24] I. Lindgren, H. Persson, S. Salomonson, L. Labzowsky, Phys. Rev. A 51 (1995) 1167.

[25] O.Yu. Andreev, L.N. Labzowsky, G. Plunien, G. Soff, Phys. Rev. A 67 (2003) 012503. 